EPJ Web of Conferences 73, 05012 (2014)

DOI: $10.1051 /$ epjconf/20147305012

(C) Owned by the authors, published by EDP Sciences, 2014

\title{
Chiral effective field theory description of the hyperon-nucleon interaction at next-to-leading order
}

\author{
S. Petschauer ${ }^{\mathrm{a}}$ \\ Physik Department, Technische Universität München, 85747 Garching, Germany
}

\begin{abstract}
In this proceeding results for hyperon-nucleon interactions calculated at nextto-leading order (i.e. one-loop order) in SU(3) chiral effective field theory are presented. The potentials include contributions from one- and two-meson exchange, and four-baryon contact terms provided by the $\mathrm{SU}(3)$ chiral Lagrangian. $\mathrm{SU}(3)$ flavor symmetry is imposed for the low-energy constants, while explicit SU(3) symmetry breaking is included only through the physical pseudoscalar-meson and baryon masses. Calculations have been performed for hyperon-nucleon scattering cross sections using a regularized LippmannSchwinger equation. A good description of the available data is achieved, comparable to modern phenomenological hyperon-nucleon interaction models. These results provide a new basis for studies of hypernuclei or hyperons in nuclear matter.
\end{abstract}

\section{Introduction}

Upcoming experimental data for hyperon-nucleon scattering will lead to new and stronger constraints on the hyperon-nucleon interaction. Moreover, because of the recently observed two solar-mass neutron stars $[1,2]$, a very stiff neutron-matter equation of state is necessary. In order to support this, strongly repulsive hyperon-nucleon forces are needed when including hyperons as additional components of the matter. Of course the hyperon-nucleon interaction is also an important input for calculations of hypernuclei.

The nucleon-nucleon interaction is well described by SU(2) chiral effective field theory [3-5]. Therefore the extension of the same scheme to SU(3) chiral effective field theory seems to be appropriate to treat strangeness and to describe the baryon-baryon interactions. Using chiral effective field theory has many advantages, for example one can improve the results by going to higher order in the smallmomentum expansion and one can derive two- and three-baryon interactions as well as few-body currents in a consistent way.

At leading order $[6,7]$ one can get already a reasonable description of the available hyperonnucleon scattering data. We have recently extended this calculation to next-to-leading order in SU(3) chiral effective field theory [8], where one obtains an improved description of the experimental data, comparable to modern phenomenological models.

\footnotetext{
${ }^{a}$ e-mail: stefan.petschauer@ph.tum.de
}

This is an Open Access article distributed under the terms of the Creative Commons Attribution License 4.0, which permits unrestricted use, distribution, and reproduction in any medium, provided the original work is properly cited. 

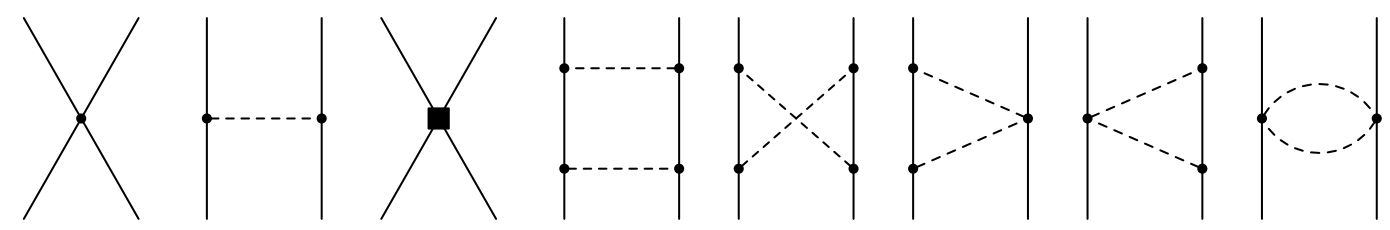

Figure 1. Feynman diagrams contributing at leading and next-to-leading order to the potential in the Weinberg power counting. Solid and dashed lines denote octet baryons and mesons, respectively. The squared box symbolizes a contact vertex proportional to $q^{2}$.

\section{Theory and results}

We use SU(3) chiral effective field theory to describe the hyperon-nucleon interactions at next-to-leading order [8]. The relevant chiral Lagrangian for the one- and two-pseudoscalar-meson-exchange potentials is the leading order meson-baryon interaction Lagrangian

$$
\mathcal{L}_{\mathrm{MB}}=\operatorname{tr}\left(\bar{B}\left(\mathrm{i} \gamma^{\mu} D_{\mu}-M_{0}\right) B\right)-\frac{D}{2} \operatorname{tr}\left(\bar{B} \gamma^{\mu} \gamma_{5}\left\{u_{\mu}, B\right\}\right)-\frac{F}{2} \operatorname{tr}\left(\bar{B} \gamma^{\mu} \gamma_{5}\left[u_{\mu}, B\right]\right),
$$

with $D_{\mu} B=\partial_{\mu} B+\left[\Gamma_{\mu}, B\right], \Gamma_{\mu}=\frac{1}{2}\left(u^{\dagger} \partial_{\mu} u+u \partial_{\mu} u^{\dagger}\right)$ and $u_{\mu}=\mathrm{i}\left(u^{\dagger} \partial_{\mu} u-u \partial_{\mu} u^{\dagger}\right)$, and where $\operatorname{tr}(.$.$) is$ the trace in flavor space. The constant $M_{0}$ denotes the baryon mass in the three-flavor chiral limit and $F$ and $D$ are axial vector coupling constants. We use the usual non-linear realization of chiral symmetry with the unitary matrix $U(x)=u^{2}(x)=\exp \left(\mathrm{i} \sqrt{2} P(x) / f_{0}\right)$, where $f_{0}$ is the pseudoscalar-meson decay constant in the chiral limit. The matrices

$$
P=\left(\begin{array}{ccc}
\frac{\pi^{0}}{\sqrt{2}}+\frac{\eta}{\sqrt{6}} & \pi^{+} & K^{+} \\
\pi^{-} & -\frac{\pi^{0}}{\sqrt{2}}+\frac{\eta}{\sqrt{6}} & K^{0} \\
K^{-} & \bar{K}^{0} & -\frac{2 \eta}{\sqrt{6}}
\end{array}\right), \quad B=\left(\begin{array}{ccc}
\frac{\Sigma^{0}}{\sqrt{2}}+\frac{\Lambda}{\sqrt{6}} & \Sigma^{+} & p \\
\Sigma^{-} & -\frac{\Sigma^{0}}{\sqrt{2}}+\frac{\Lambda}{\sqrt{6}} & n \\
-\Xi^{-} & \Xi^{0} & -\frac{2 \Lambda}{\sqrt{6}}
\end{array}\right)
$$

collect the pseudoscalar mesons and octet baryons.

The Weinberg power counting scheme is applied to the potential, i.e. the two-particle irreducible part of the T-matrix [7]. This potential is iterated to all orders by the Lippmann-Schwinger equation. The potentials at leading order are obtained from one-meson exchange diagrams and short-range nonderivative four-baryon contact terms, cf. Fig. 1. At next-to-leading order there arise two-meson exchange diagrams at the one-loop level, as shown in Fig. 1. The corresponding results are available in analytical form. For the different two-baryon channels the expressions vary only by an SU(3) factor and the masses of the exchanged mesons. Additionally at next-to-leading order contact terms with two powers of momenta as well as contact terms proportional to quark masses contribute [9]. The latter lead to an explicit breaking of SU(3) symmetry due to the different masses of the up, down and strange quarks. However, because of the shortage of experimental data, these contributions have been omitted and only SU(3) symmetric low-energy constants have been used. Also the antisymmetric spin-orbit term leading to spin singlet-triplet mixing has been omitted for the same reason. SU(3) breaking is only included by the physical masses of the exchanged mesons $(\pi, K, \eta)$ and baryons.

With the potential projected into partial waves the Lippmann-Schwinger equation reads

$$
T_{v^{\prime \prime} v^{\prime}}^{\rho^{\prime \prime} \rho^{\prime}, J}\left(p^{\prime \prime}, p^{\prime} ; \sqrt{s}\right)=V_{v^{\prime \prime} v^{\prime}}^{\rho^{\prime \prime} \rho^{\prime}, J}\left(p^{\prime \prime}, p^{\prime}\right)+\sum_{\rho, v} \int_{0}^{\infty} \frac{d p p^{2}}{(2 \pi)^{3}} V_{v^{\prime \prime} v}^{\rho^{\prime \prime} \rho, J}\left(p^{\prime \prime}, p\right) \frac{2 \mu_{v}}{q_{v}^{2}-p^{2}+i \eta} T_{\nu v^{\prime}}^{\rho \rho^{\prime}, J}\left(p, p^{\prime} ; \sqrt{s}\right),
$$


$\Lambda p->\Lambda$

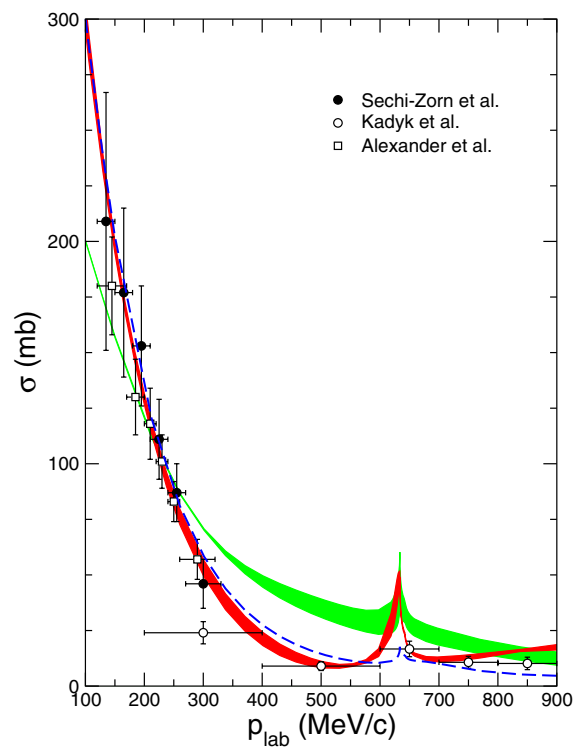

$\Lambda \mathrm{p}^{1} \mathrm{~S}_{0}$

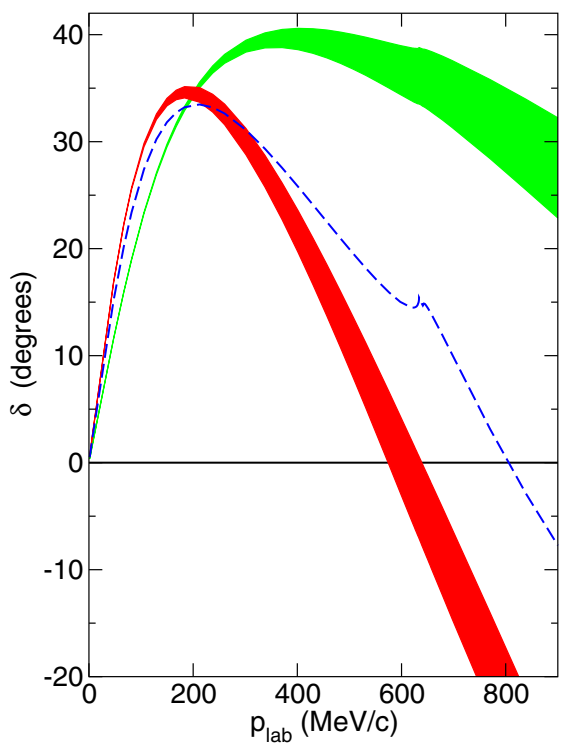

Figure 2. Total cross section and the ${ }^{1} S_{0}$ phase shift for the $\Lambda N$ interaction. Shown are the results of the leading order (green/light band) [7] and next-to-leading order (red/dark band) [8] calculation in SU(3) chiral effective field theory and of the Jülich '04 meson exchange model (blue dashed line) [10].

where $v$ labels a particle channel and $\rho$ a partial wave and $q_{v}$ is the on-shell momentum. In order to obtain the T-matrix this coupled-channel Lippmann-Schwinger equation is solved numerically. An exponential regulator function is used to remove high energy components of the potential. The Coulomb interaction is included by the Vincent-Phatak method. A simultaneous description of the sectors with strangeness 0 ( $N N$ scattering) and -1 was not possible. Therefore the $\mathrm{SU}(3)$ symmetric low-energy constants were determined by fitting to experimental data with strangeness -1 only.

As examples for our results we show the total cross section and the ${ }^{1} S_{0}$ phase shift for the $\Lambda N$ interaction in Fig. 2. Although the leading order approach gives already a reasonable fit of the experimental data, we can describe the experimental data much better in the next-to-leading order calculation and come closer to the phenomenological model Jülich '04. The cutoff dependence introduced by the regulator function is also reduced, as can be seen from the narrower bands at NLO. The ${ }^{1} S_{0} \Lambda N$ phase shift at next-to-leading order has a stronger downward bend in comparison to the leading order result and the phenomenological Jülich '04 model. This points to moderate attraction at low momenta and strong repulsion at higher momenta. Such a feature agrees with the expectation that a repulsive $\Lambda N$ interaction is necessary to obtain an equation of state which supports two-solar-mass neutron stars. Therefore calculations of neutron stars with the present chiral hyperon-nucleon potential should be performed.

\section{Summary and outlook}

We have used SU(3) chiral effective field theory in the heavy baryon approach to calculate the hyperonnucleon interaction at next-to-leading order [8]. We have obtained a good description of the available hyperon-nucleon scattering data, comparable to modern phenomenological models. The $\Lambda N$ interaction is found to be repulsive at higher momenta. This could provide a stiff enough equation of state to support 


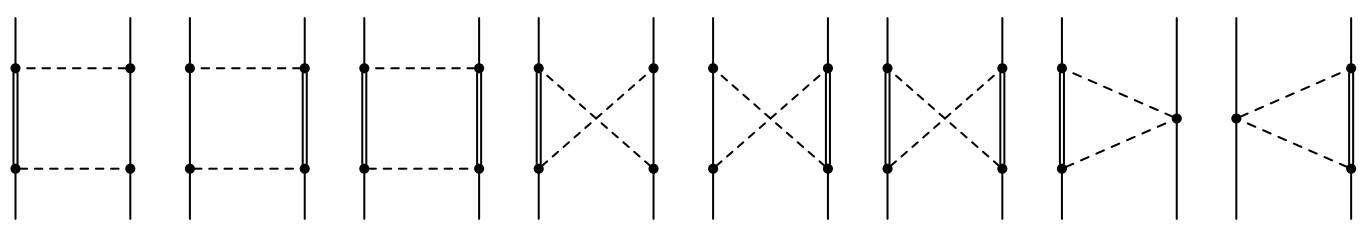

Figure 3. Additional contributions at next-to-leading order to the potential with the inclusion of decuplet baryons. Double lines denote decuplet baryons.

two-solar-mass neutron stars with hyperons. Another source for repulsion could come from three-baryon forces [11], similar to the nuclear forces [12]. Calculations of the leading three-baryon forces in chiral effective field theory are in progress [13].

The two-pion exchange nucleon-nucleon interaction at $\mathrm{N}^{2} \mathrm{LO}$ involves large low-energy constants which originate primarily from the strongly coupled $\pi N \Delta$ system. By including the delta isobar as an explicit degree of freedom these large contributions get promoted to next-to-leading order in the hierarchy of $N N$ interaction terms [4, 12]. It would therefore be interesting to include decuplet baryons $\left(\Delta, \Sigma^{*}, \Xi^{*}, \Omega\right)$ in our next-to-leading order calculation as well [14], such that potentially large (not yet considered) $\mathrm{N}^{2} \mathrm{LO}$ effects get also promoted to next-to-leading order, cf. Fig. 3.

I would like to thank the organizers of MENU 2013 for the invitation to this conference. This work has been supported in part by DFG and NSFC (CRC110). I thank J. Haidenbauer, N. Kaiser, U.-G. Meißner, A. Nogga and $\mathrm{W}$. Weise for collaborating on the topic covered by my talk.

\section{References}

[1] P.B. Demorest et al., Nature 467, 1081 (2010)

[2] J. Antoniadis et al., Science 340, 6131 (2013)

[3] E. Epelbaum, W. Glöckle, U.-G. Meißner, Nucl. Phys. A 747, 362 (2005)

[4] R. Machleidt, D. Entem, Phys. Rept. 503, 1 (2011)

[5] J.W. Holt, N. Kaiser, W. Weise, Prog. Part. Nucl. Phys. 73, 35 (2013)

[6] M.J. Savage, M.B. Wise, Phys. Rev. D 53, 349 (1996)

[7] H. Polinder, J. Haidenbauer, U.G. Meißner, Nucl. Phys. A 779, 244 (2006)

[8] J. Haidenbauer, S. Petschauer, N. Kaiser, U.-G. Meißner, A. Nogga, W. Weise, Nucl. Phys. A 915, 24 (2013)

[9] S. Petschauer, N. Kaiser, Nucl. Phys. A 916, 1 (2013)

[10] J. Haidenbauer, U.-G. Meißner, Phys. Rev. C 72, 044005 (2005)

[11] D. Lonardoni, S. Gandolfi, F. Pederiva, Phys. Rev. C 87, 041303 (2013)

[12] E. Epelbaum, H.-W. Hammer, U.-G. Meißner, Rev. Mod. Phys. 81, 1773 (2009)

[13] S. Petschauer, arXiv:1312.4467 (2013)

[14] S. Petschauer, Nucl. Phys. A 914, 238 (2013) 